\title{
Phenytoin Toxicity Presenting as Encephalopathy With Fatal Outcome: A Case Report
}

\author{
Bhawna Sharma ${ }^{\mathrm{a}}$, Rahul Handa ${ }^{\mathrm{a}}$, Swayam Prakash ${ }^{\mathrm{a}, \mathrm{b}}$, \\ Kadam Nagpal ${ }^{a}$, Pankaj Gupta ${ }^{\mathrm{a}}$
}

\begin{abstract}
Phenytoin is the oldest sedative antiepileptic drug and one of the most widely prescribed antiepileptic drug effective against all types of partial and tonic-clonic seizures except in absence seizures. Medication errors with phenytoin often occur and can be lifethreatening but have rarely been fatal, especially in adults. We here report a fatal case of phenytoin-induced encephalopathy in an adult due to inadvertent drug overdose and highlight the importance of clinicians educating the patients and caregivers regarding the toxic manifestations of phenytoin, so that toxicity can be recognized early and delay in therapeutic intervention can be avoided.
\end{abstract}

Keywords: Encephalopathy; Phenytoin toxicity; Overdose; Fatal case

\section{Introduction}

Poisoning is a global health hazard. In the year 2004, as per WHO database, approximately 346,000 patients died worldwide from unintentional poisoning. Among these $91 \%$ death occurred in low- and middle-income economic countries [1]. Introduced in 1938, phenytoin is the oldest sedative antiepileptic drug and one of the most widely prescribed antiepileptic drug effective against all types of partial and tonic-clonic seizures except in absence seizures. However, it requires regular therapeutic drug monitoring due to its narrow therapeutic range. It is relatively safe when its blood level is maintained within the therapeutic range $[2,3]$. Phenytoin follows a non-linear kinetics and even a minor increase in the dose

Manuscript accepted for publication November 28, 2013

${ }^{\mathrm{a}}$ Department of Neurology, SMS Medical College, Jaipur, Rajasthan, India

${ }^{\mathrm{b}}$ Corresponding author: Swayam Prakash, Department of Neurology, SMS Medical College, Jaipur, Rajasthan, India.

Email: vanswayam@rediffmail.com

doi: http://dx.doi.org/10.4021/jnr249w may lead to toxic concentrations. However, overdose and medication errors are the more common causes of its toxicity [3]. Medication errors with phenytoin often occur and can be life-threatening but have rarely been fatal, especially in adults. We here report a fatal case of phenytoin-induced encephalopathy in an adult due to inadvertent drug overdose.

\section{Case Report}

A 35-year-old female presented to the emergency department with acute onset of altered sensorium since three days. Patient was a known case of seizure disorder which was under control with oral phenytoin (100 mg three times a day) since three years. She did not have any history of recent fever or trauma or any recurrence of seizure. On physical examination, she was afebrile with normal pulse rate and blood pressure. On neurologic examination, her Glasgow coma scale was E2M4V2; she had nystagmus on lateral gaze, deep tendon reflexes were depressed, and had ataxia. There were no meningeal signs of irritation or focal neurologic deficits.

Routine investigations, complete blood count, comprehensive metabolic panel (including renal and liver function tests), chest X-ray (per abdomen view), electrocardiography, arterial blood gas analysis and ultrasonography abdomen were all normal. Cerebrospinal fluid examination and magnetic resonance imaging brain with contrast were normal. Electroencephalography showed generalized slowing without any epileptiform discharge.

Her medications were re-evaluated and it was found that, by mistake, the patient had been taking $300 \mathrm{mg}$ thrice daily dose of phenytoin since past one week instead of prescribed $100 \mathrm{mg}$ three times a day. The patient was dispensed $300 \mathrm{mg}$ tablets instead of prescribed $100 \mathrm{mg}$ tablets. Her blood sample was sent for phenytoin level and showed a phenytoin level of $144 \mu \mathrm{g} / \mathrm{mL}$. Patient was diagnosed as a case of phenytoin-induced encephalopathy and drug toxicity. Phenytoin was immediately stopped and supportive therapy was initiated. Despite of best in hospital care, her condition deteriorated and respiratory depression ensued and she was comatose on hospital day 3. She was put on ventilator but she died due to generalized neurologic depression and respi- 
ratory failure on hospital day 7 .

\section{Discussion}

Phenytoin toxicity can be precipitated by acute overdose, inadvertent toxicity from chronic use, altered physiology (pregnancy or various disease states), inter-individual variability, or drug interactions. Acute ingestion of more than 20 $\mathrm{mg} / \mathrm{kg}$ usually results in clinical toxicity. Inadvertent toxicity occurs due to errors in prescribing, dispensing, or administration; increases in doses or changes in brands or formulations of the drug [4]. In our country, the medication error and drug dispensing error occur due to availability of two strengths of phenytoin (100 mg and $300 \mathrm{mg}$ ). Oral phenytoin overdoses in adults are rarely severe or life-threatening and very unusually lead to fatal neurologic complications. The "2011 Annual Report of the American Association of Poison Control Centers National Poison Data System (NPDS): 29th Annual Report" reports 3,173 cases of phenytoin toxicity but lists only one case as fatal [5].

There are many clinical conditions which influence phenytoin metabolism and can lead to high phenytoin levels and toxicity. Toxicity depends on the route and duration of exposure, dosage, pharmacogenetic variations (mutations in cytochrome P450 enzyme), age group (elderly more susceptible), hepatic and renal impairment and black ethnicity $[4,6]$. Target organ damage usually depends on route and duration of exposure. When IV fosphenytoin (water soluble prodrug of phenytoin for IV use) is administered at an excessive rate, cardiac toxicity is usually encountered, most notable toxic signs being cardiac arrhythmias, with or without hypotension, and/or central nervous system (CNS) depression. Acute oral overdosage results primarily in CNS toxicities damages cerebellum and vestibular system; high doses have been associated with marked cerebellar atrophy. Toxic effects associated with chronic treatment also are primarily dose-related cerebellar-vestibular effects and also include other CNS effects, behavioral changes, increased seizures frequency, gastrointestinal symptoms, hirsutism, gingival hyperplasia, osteomalacia and megaloblastic anemia. Usually, these effects can be reduced by proper dose adjustment [6].

Metabolic pathway of phenytoin plays a crucial role in its toxicity. It is primarily metabolized by parahydroxylation in the liver followed by glucoronic acid conjugation. The hydroxylating enzyme saturates at therapeutic doses, and the metabolism follows first order kinetics at low doses but changes to zero order kinetics once the hydroxylating enzyme becomes saturated [3]. The plasma half-life of phenytoin ranges between 6 and $24 \mathrm{~h}$ at plasma concentrations $<10 \mathrm{mcg} / \mathrm{mL}$ but increases with higher concentrations while toxic effects such as nystagmus develop at concentrations around $20 \mu \mathrm{g} / \mathrm{mL}$. Deaths have been reported at levels of 50 $70 \mu \mathrm{g} / \mathrm{mL}$ [6]. The phenytoin level in our patient was $144 \mu \mathrm{g} /$
$\mathrm{mL}$ which is far greater than the toxic range. The patient was taking three times the prescribed dose since one week which resulted in accumulation of phenytoin and its toxic effects.

Phenytoin toxicity usually presents early and is detectable by its manifestations of ataxic gait, nystagmus and mental status changes [7]. Our patient had altered sensorium since three days, but due to unawareness at the level of caregiver, timely interventions could not be instituted. Due to delay in recognition of toxicity by the patient/caregiver and continued exposure to high dose of phenytoin, our case had fatal outcome. Fatal outcome has rarely been reported with single drug toxicity of phenytoin. Mellick et al, in a literature article, had presented a review of published cases of fatal phenytoin toxicity. Most of the fatal cases in their review were concerned with children or were cases of mixed overdose [8].

Treatment recommendations beyond supportive care are unclear. Poddutoor et al recommended charcoal hemoperfusion in their case report [9], while Sen et al had recommended molecular adsorbent recirculating system [10]. None of these drug elimination technique have proven benefit. Our patient was given supportive therapy, but due to severity of the overdose, she could not survive.

\section{Conclusion}

This case highlights the need of awareness and education at the level of patients and caregivers. We recommend that the clinicians should educate the patients and caregivers regarding the toxic manifestations of the drug, so that toxicity can be recognized early and delay in therapeutic intervention can be avoided. Whenever possible, change in brand and dose strength should be avoided, to decrease unnecessary confusion at the level of patient. Cautions should be exercised at the level of pharmacist and while dispensing the drug, they should be more vigilant at the prescribed strength. Lastly, this case reminds the clinicians about the need of regular follow-up and monitoring of serum phenytoin level even when the seizure is well under control.

\section{References}

1. Shobha C, Ramesh M, Parthasarathi G. Poison information center- an overview of its significance, organization and functioning. IJOPP. 2011;4(4):14-20.

2. Porter RJ, Beldrum BS. Antiseizure Drugs (chapter 24). In: Katzung BG, editor. Basic and Clinical Pharmacology. 11th edition. New York (NY): McGraw-Hill; 2009 (Access medicine).

3. Akula R, Hasan S, Pipalla R, Ferguson C. Noncompliance leading to drug accumulation resulting in phenytoin toxicity. J Natl Med Assoc. 2003;95(12):1201-1203. 
4. Craig S. Phenytoin poisoning. Neurocrit Care. 2005;3(2):161-170.

5. Bronstein AC, Spyker DA, Cantilena LR, Jr., Rumack BH, Dart RC. 2011 Annual report of the American Association of Poison Control Centers' National Poison Data System (NPDS): 29th Annual Report. Clin Toxicol (Phila). 2012;50(10):911-1164.

6. McNamara JO: Pharmacotherapy of the epilepsies (Chapter 19). In: Brunton LL, editor. Goodman and Gilman's Pharmacological Basis of Therapeutics. 11th edition. New York (NY): McGraw-Hill; P 321-338.

7. Su CM, Kung CT, Wang YC, Lu CH. Life-threatening cardiotoxicity due to chronic oral phenytoin overdose. Neurol India. 2009;57(2):200-202.

8. Mellick LB, Morgan JA, Mellick GA. Presentations of acute phenytoin overdose. Am J Emerg Med. 1989;7(1):61-67.

9. Kumar PP, Lingappa L, Shah MA, Shaikh FA. Charcoal hemoperfusion for phenytoin intoxication. Indian Pediatr. 2012;49(2):152-153.

10. Sen S, Ratnaraj N, Davies NA, Mookerjee RP, Cooper CE, Patsalos PN, Williams R, et al. Treatment of phenytoin toxicity by the molecular adsorbents recirculating system (MARS). Epilepsia. 2003;44(2):265-267. 